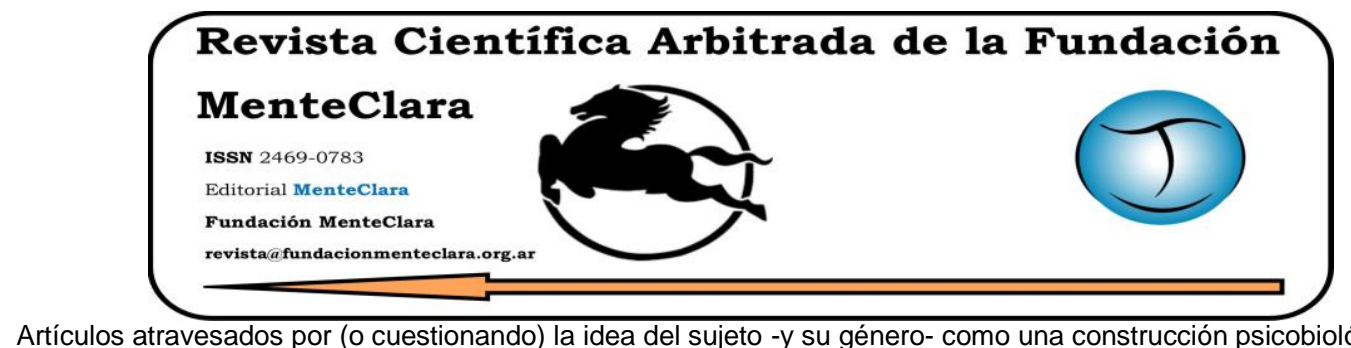

Artículos atravesados por (o cuestionando) la idea del sujeto -y su género- como una construcción psicobiológica de la cultura. Articles driven by (or questioning) the idea of the subject -and their gender- as a cultural psychobiological construction

Vol. 2 (2), 2017

ISSN 2469-0783

https://datahub.io/dataset/2017-2-2-e32

\title{
RESILIENCIA Y ESPIRITUALIDAD. APORTES PARA SU ESTUDIO DESDE UNA PERSPECTIVA PSICOLÓGICA
}

\author{
RESILIENCE AND SPIRITUALITY. CONTRIBUTIONS TO STUDY THEM FROM A \\ PSYCHOLOGICAL PERSPECTIVE
}

Juan Ignacio Irurzun ${ }^{1}$, Joaquin Mezzadra², Maximiliano Preuss ${ }^{3}$

Cómo citar este artículo / Citation: Irurzun J. I., Mezzadra J., Preuss M. (2017). "Resiliencia y espiritualidad. Aportes para su estudio desde una perspectiva psicológica". Revista Científica Arbitrada de la Fundación MenteClara, 2(2), 205-216. DOI: $10.32351 /$ rca.v2.2.34

Copyright: (C) 2017 RCAFMC. Este artículo de acceso abierto es distribuido bajo los términos de la licencia Creative Commons Attribution-Non Commercial (by-cn) Spain 3.0. Recibido: 01/10/2017. Aceptado: 25/10/2017 Publicación online: $30 / 10 / 2017$

Conflicto de intereses: Ninguno que declarar.

Este trabajo fue patrocinado en en forma conjunta por la Universidad de Flores, la Universidad de Buenos Aires y por la Universidad Abierta Interamericana.

\section{Resumen}

En la actualidad, distintos autores han sugerido que el concepto de resiliencia se encuentra asociado a la espiritualidad, de modo que aquellas personas que se consideran espirituales podrían presentar características resilientes (Poseck, Baquero \& Jiménez, 2006; Girard, 2007). Sin embargo, son pocos los trabajos que se proponen realizar una revisión sistemática de la literatura en relación al vínculo entre ambos constructos. Por este motivo, el presente trabajo se propone realizar una búsqueda bibliográfica en bases de datos - SCIELO, LATINDEX, entre otras - con el

\footnotetext{
${ }^{1}$ Universidad de Flores (UFLO), Argentina. juanignacio.irurzun@uflo.edu.ar

2 Universidad de Buenos Aires (UBA), Argentina.

${ }^{3}$ Universidad Abierta Interamericana (UAI), Argentina.
} 
objeto de revisar críticamente los hallazgos empíricos reportados por los diferentes estudios. Se concluye que, si bien se han incrementado la cantidad de trabajos en el área en los últimos años, aún se requiere un mayor número de investigaciones, particularmente en el contexto latinoamericano. A la vez, la definición y evaluación de la espiritualidad ha presentado considerables dificultades, por lo que aún se precisa mejorar las herramientas disponibles para su indagación empírica.

\begin{abstract}
Currently, different authors have suggested that the concept of resilience is associated with spirituality, so that those who consider themselves spiritual could have resilient characteristics (Poseck, Baquero \& Jiménez, 2006, Girard, 2007). However, there are few works that propose to perform a systematic review of the literature in relation to the link between both constructs. For this reason, the present work intends to carry out a bibliographic search in databases - SCIELO, LATINDEX, among others - in order to review critically the empirical findings reported by the different studies. It is concluded that, although the number of jobs in the area has increased in the last few years, it is still necessary to increase the amount of research, particularly in the Latin American context. At the same time, the definition and evaluation of spirituality has presented considerable difficulties, so it is still necessary to improve the tools available for its empirical investigation.
\end{abstract}

\title{
Palabras Claves/ Keywords
}

Espiritualidad; Resiliencia; Psicología; Spirituality; Resilience; Psychology 


\section{Introducción.}

El fenómeno espiritual ha sido considerado un problema de marcado interés en la literatura psicológica. Sin embargo no es hasta mediados de la década del ochenta que la cantidad de estudios específicos vinculados a temáticas religiosas ha crecido lo suficiente como para promover el desarrollo del área específica de la psicología de la religión y de la espiritualidad. En este contexto, distintos autores se han propuesto explorar el modo en que la espiritualidad y la religiosidad se encuentran asociados a la resiliencia. Así, distintos estudios se ocupan de identificar los aspectos específicos de los constructos numinosos que se asocian a una mayor o menor capacidad de afrontar distintas situaciones problemáticas. Debido a que no se han relevado antecedentes que se propongan realizar una revisión sistemática de la literatura en el contexto local, el presente trabajo tiene por objetivo revisar criticamente los hallazgos empíricos reportados por las investigaciones actuales en el contexto internacional.

\section{La espiritualidad desde una perspectiva psicológica}

Si bien el carácter espiritual tuvo relevancia desde los primeros inicios de la psicología (Allport, 1950; James, 1902), fue a partir de las introducciones que hizo en su trabajo Gorsuch (1984) que la cantidad de artículos publicados y el número de revistas especializadas en el tema se han incrementado (Belzen \& Hood, 2006; Jacob A Belzen, 2015; Muñoz, 2004). A la vez, durante los últimos veinte años el número de estudios que centran su atención en el papel que ocupa la espiritualidad en el afrontamiento de estresores vitales ha crecido exponencialmente (Ano \& Vasconcelles, 2005; Pargament, 1997; Phelps et al., 2009). Diferentes autores han observado que algunas personas se apoyan en la religión y 
en la espiritualidad como un recurso para comprender y afrontar momentos dificiles (Abu-Raiya \& Pargament, 2015; Ahles, Mezulis, \& Hudson, 2016; Feder et al., 2013). Etimológicamente, la palabra espiritualidad surge en el occidente cristiano a inicios del primer milenio, a partir del término latino spiritualitas, proveniente del sustantivo spiritus (espíritu) y del adjetivo spiritual (espiritual), traducciones de los términos griegos pneuma (soplo), y pneumatké, es decir, etéreo o no material (Príncipe, 1983). Desde ya, estudiar la espiritualidad en los individuos es considerado un aporte de relevancia para la psicología, dado que el sentido de la vida constituye un tema central en el desarrollo de la personalidad. Si bien no se identifica una concepción univoca, según Piedmont (2001), la espiritualidad es definida como una disposición inherente a los humanos con las características de ser universal e integradora, que tiene como meta orientar la conducta permitiendo trascender a la existencia cercana con un propósito y un sentido de vida más amplio, relativo al destino del hombre después de su muerte. De acuerdo con este autor, ante la inminencia del conocimiento de que en algún momento su cuerpo va a perecer, las personas necesitan construir un sentido más amplio para la vida que llevan (Piedmont, 2004). Desde este análisis, las mismas se insertan dentro de una unidad fundamental encontrando una conexión con el prójimo que no puede ser obstruida ni por el fallecimiento. Siguiendo las sugerencias del autor, distintos estudios han explorado el constructo desde el modelo de los cinco factores de la personalidad (Piedmont, 1999; Simkin, 2017).

Por otra parte, asociado al constructo de la espiritualidad existe lo que Pargament (1997) define como el afrontamiento religioso, es decir, un proceso orientado y contextual que pone a la religión como el eje central en el desarrollo de estrategias de resolución de conflictos y problemas. A su vez, el autor identifica patrones positivos y negativos de afrontamiento 
religioso para distinguir dos orientaciones que prevalecen en el individuo al momento de recurrir a la religión como estrategia de afrontamiento: mientras que los patrones positivos reflejan una relación segura con una fuerza trascendente, un sentido de conexión espiritual con los demás, y una visión benevolente del mundo; los negativos, por el contrario, reflejan tensiones espirituales subyacentes, descontento espiritual y luchas dentro de uno mismo, con los demás y con lo divino. El estudio del afrontamiento religioso representa una de las lineas de investigación que mayor interés ha cobrado tanto en el contexto internacional como en el plano local (Mezzadra \& Simkin, 2017; Pargament 1997). Diversos estudios indican que las personas que viven su religión de manera positiva, generando vínculos de confianza con Dios, tienden a sobreponerse a diversos estresores con mayor facilidad que aquellas que experimentan una lucha interna constante con Dios o con su comunidad religiosa (Bryant-Davis et al., 2015; Lee, Nezu, \& Nezu, 2014; Rosmarin, Bigda-Peyton, Öngur, Pargament, \& Björgvinsson, 2013; Vallurupalli et al., 2012).

Cabe señalar que en la literatura especializada suelen distinguirse los términos espiritualidad y religiosidad. De este modo, mientras que la espiritualidad es considerada como una motivación que orienta el comportamiento del individuo en el esfuerzo de construir un sentido más profundo para su vida desde una perspectiva escatológica (relativa al ser humano luego de su muerte), la religiosidad refiere al modo en que la propia espiritualidad es moldeada y la forma en que se expresa a través de una organización comunitaria o social (Piedmont, 2012). 


\section{La resiliencia desde una perspectiva psico-social}

En la actualidad existen numerosas investigaciones que arribaron a una definición del término resiliencia. Mientras algunos autores la describen desde una mirada intrapersonal resaltando los aspectos de la persona, otros ponen el foco en lo interpersonal y la relevancia con su contexto social más próximo. Si bien no existe una concepción única, hablar de resiliencia hoy es hablar de un constructo teórico empleado dentro de disciplinas tales como la psicología, sociología, antropología, salud, trabajo social, economía y filosofia. Todas ellas tratan de estudiar la resiliencia desde la infancia, pasando por la adolescencia hasta llegar a la edad adulta.

Es importante destacar que la asociación o agrupamiento del término con otros conceptos han dado lugar, en más de una ocasión, a erróneas interpretaciones del mismo, tales como: sentido del humor, control personal, persona "resistente", empatía, en otras. (Earvolino-Ramirez, 2007; Fernández-Lansac y Crespo, 2011; Garcia-Ona, Jakobowski, \& O'Flagerty, 2013; Windle, 2011). La palabra resiliencia proviene del latín resilio y es utilizada en el campo de la física para describir el proceso por el cual un material vuelve a su estado anterior, esto significa volver, rebotar, saltar hacia atrás, ser repelido o surgir (Muller, 2008; Diccionario de la Real Academia de la Lengua Española, 2005). Posteriormente, el término fue adaptado a las ciencias sociales para describir a las personas que, a pesar de nacer y vivir en situaciones de alto riesgo, logran desarrollarse psicológicamente sanas y exitosas (Machuca, 2002).

Contemporáneamente, se asegura que se trata de un suceso psicológico y es utilizado en diferentes disciplinas incluida la medicina (Cornejo, 2010). Si bien es un concepto homogéneo, se emplea en el campo de la psicología como adaptación efectiva cuando un sujeto 
atraviesa condiciones de peligro (Gaxiola, 2013). En este sentido, se entiende la resiliencia como un constructo dinámico que incluye una amplia clase de fenómenos implicados en las adaptaciones exitosas en el contexto de amenazas significativas para el desarrollo (Villalba, 2003).

Poseck, Baquero y Jiménez (2006) señalan que en los primeros antecedentes de psicología sobre la temática, existía un anclaje de la misma en la descripción de conductas individuales de superación, las cuales parecían ser casos puntuales y anecdóticos. Sin embargo, estudios posteriores han demostrado que la resiliencia es un proceso más amplio en el cual intervienen patrones y características generales del individuo. La resiliencia, a pesar de que requiere una respuesta subjetiva, no es una característica singular, pues está condicionada tanto por factores individuales como socio-ambientales, emergiendo de una gran heterogeneidad de influencias ecológicas que confluyen para producir una reacción excepcional frente a una amenaza importante.

Desde esta visión más amplia, se ha entendido a la resiliencia como el resultado de la combinación o interacción entre los atributos del individuo (internos) y su ambiente familiar, social y cultural (externos) que posibilitan superar el riesgo y la adversidad de forma constructiva. En linea con esta perspectiva, Grotberg (2004) la describe como un llamado a centrarse en cada sujeto como alguien auténtico y genuino, resaltando las capacidades $\mathrm{y}$ habilidades personales que surgieron durante alguna sopesada situación que haya puesto en riesgo su persona, y ya transcurrida ésta, logre revestir las actitudes más provechosas logrando un fortalecimiento contra amenazas posteriores. Asimismo, Morera (2007) la define como la habilidad que tiene una persona, sistema u organización para resistir circunstancias conflictivas preservando la integridad del organismo, reaccionando favorablemente a las dificultades y tratando de salir reforzada de ellas. Por último, desde 
una perspectiva más trascendental, Krauskopf (2007) la define como la composición de aptitudes y acciones destinadas a encontrar el sentido de la vida y el progreso frente a la adversidad.

\section{Asociaciones entre resiliencia y espiritualidad en la literatura especializada}

Dado que el sentido de la vida humana es un componente que puede ligarse intrínsecamente a la resiliencia, existen autores que la vinculan con el concepto de espiritualidad (Poseck, Baquero \& Jiménez, 2006; Girard, 2007). Las investigaciones en el área han hecho esfuerzos buscando una verificación de su asociación en distintas poblaciones, las cuales han vivido situaciones movilizantes, generalmente pérdidas, abusos o enfermedades que afectaron la sensibilidad de las personas enfrentándolas a la posibilidad real y próxima de la muerte, constituyéndose en eventos centrales para la formación y el desarrollo de su identidad (Matrángolo, Paz, \& Simkin, 2015; Navas \& Villegas, 2007). De acuerdo con el rastreo del estado del arte, la espiritualidad es considerada como facilitadora de la recuperación personal ante distintas enfermedades, a la vez que promueve mayor bienestar psicológico (Sanchez, 2004; Simkin, 2016). Aunque en las revisiones de la literatura especializada se destaca que la espiritualidad suele considerarse como un constructo universal en todos los individuos (Piedmont, 1999; Simkin \& Cermesoni, 2014), cada sujeto podría poseer diferentes niveles de conciencia sobre sus capacidades, por lo tanto, sus efectos sobre la resiliencia podrian variar según cada persona, dependiendo de su personalidad, su contexto y la etapa en su ciclo vital (Laspina, 1996). La espiritualidad genera un alto nivel de trascendencia posibilitando un propósito a la existencia misma y puede ser expandido en sentidos escatológicos. Es esta misma persecución hacia un significado existencial 
la que genera un estado de bienestar subjetivo con estándares que favorecen la resiliencia individual (Rodriguez, 2011).

De acuerdo con la literatura, la religiosidad, por su parte, se encuentra asociada de manera tanto positiva como negativa a la salud mental (Simkin \& Azzollini, 2015). Desde una perspectiva psicológica, ésta ha sido comprendida como un modelo mental genérico que incide en la evaluación y en el procesamiento de la información, de modo que, de acuerdo a diferentes factores como los rasgos de la personalidad, estas evaluaciones podrian afectar el modo en que las personas significan sus propias experiencias (James, \& Wells, 2003; Simkin \& Etchevers, 2014). Tal es así que la religiosidad promueve en el individuo la posibilidad de desarrollar un afrontamiento donde se utilizan creencias y comportamientos religiosos para prevenir y/o aliviar las consecuencias negativas de sucesos de vida estresantes, tanto como para facilitar la resolución de problemas (Pargament, 1997).

\section{Conclusión}

Si bien tanto la espiritualidad como la religiosidad han sido conectadas al fenómeno de la resiliencia, aún se requiere continuar incrementando la cantidad de estudios que puedan aportar mayor fortaleza teórica a la naturaleza de estas asociaciones. A la vez, resulta necesario continuar trabajando en las definiciones conceptuales de los términos en los que se describen los fenómenos religiosos y espirituales desde una perspectiva psicológica. Tales precisiones podrian contribuir a generar un mayor consenso para la evaluación empírica de los constructos numinosos, lo que podría generar un impacto en los resultados de las investigaciones

que se propongan explorar sus asociaciones. Por último, considerar la espiritualidad y la religiosidad en el marco del modelo de los cinco 
factores de la personalidad, podría contribuir a identificar aspectos de las creencias espirituales y religiosas que se asocian con una mayor o menor resiliencia y, por tal motivo, con una mayor o menor salud mental.

\section{Referencias}

Abu-Raiya, H., \& Pargament, K. I. (2015). Religious coping among diverse religions: Commonalities and divergences. Psychology of Religion and Spirituality, 7(1), 24-33. doi: $10.1037 / \mathrm{a} 0037652$

Allport, G. W. (1950). The individual and his religion. New York: Macmillan Company.

Ano, G. G., \& Vasconcelles, E. B. (2005). Religious coping and psychological adjustment to stress: A meta-analysis. Journal of Clinical Psychology, 61(4), 461-480. doi: $10.1002 /$ jclp.20049

Belzen, J. A. (2015). Infrastructure in Early Psychology of Religion: The Fate of the First European Journals. International Psychology, Practice and Research, 5, 1-16.

Belzen, J. A., \& Hood, R. W. (2006). Methodological issues in the psychology of religion: toward another paradigm? The Journal of Psychology, 140(1), 5-28. http://doi.org/10.3200/JRLP.140.1.5-28

Bryant-Davis, T., \& Wong, E. C. (2013). Faith to move mountains: Religious coping, spirituality, and interpersonal trauma recovery. American Psychologist, 68(8), 675-684. doi: $10.1037 / \mathrm{a} 0034380$

Cornejo, W. (2010). Resiliencia: Una definición. Revista Electrónica Athenea, 2. (Recuperado el 27 de abril de 2017, de http://www.academiaperuanadepsicologia.org)

Earvolino-Ramirez, M. (2007). Resiliencie: A conceptual analysis. Nursing Forum, 41, 73-82

Feder, A., Ahmad, S., Lee, E. J., Morgan, J. E., Singh, R., Smith, B. W. ... Charney, D. S. (2013). Coping and PTSD symptoms in Pakistani earthquake survivors: Purpose in life, religious coping and social support. Journal of Affective Disorders, 147(1-3), 156-163. doi: $10.1016 / j . j a d .2012 .10 .027$

Fernández-Lansac, V. y Crespo, M. (2011). Resiliencia, personalidad resistente y crecimiento en cuidadores de personas con demencia en el entorno familiar: Una revisión. Clínica y Salud, 22, 21-40. DOI: 10.593/c12011v22n1a2.

Garcia-Dia, M. J., DiNapoli, J. M., Garcia-Ona, L., Jakobowski, R., \& O'Flaherty, D. (2013). Conceptual analysis: Resiliencie. Archives of Psychiatric Nursing 27, 264-270. DOI: 10.1016/j.apnu.2013.07.003.

Gaxiola, J. C. (2013). Aportaciones conceptuales al estudio de la resiliencia. En J. C. Gaxiola y J. Palomar (Coords.), Estudios de resiliencia en América Latina. Volumen 2 (pp. 1-17). México: Universidad de Sonora/Universidad Iberoamericana/Pearson. 
Girard, G. (2007). La espiritualidad: ¿promueve la resiliencia?. En M. Munist, N. Suárez Ojeda, D. Krauskopf \& T. Silber (Comp.), Adolescencia y resiliencia (pp.139-151). Buenos Aires: Paidós.

Gorsuch, R. L. (1984a). Measurement: The boon and bane of investigating religion. American Psychologist, 39(3), 228-236. http://doi.org/10.1037/0003-066X.39.3.228

Grotberg, E. (2004). Adolescentes contra la violencia: el poder de la resiliencia. En A. Melillo, E. Suárez Ojeda \& D. Rodríguez (Comp.), Resiliencia y subjetividad: los ciclos de la vida (pp.155-171). Buenos Aires: Paidós.

James, A., \& Wells, A. (2003). Religion and mental health: towards a cognitive behavioural framework. British Journal of Health Psychology, 8(3), 359-376. doi:10.1348/135910703322370905

James, W. (1902). The varieties of religious experience. (1994th ed.). New York: The Modern Library.

Krauskopf, D. (2007). Sociedad, adolescencia y resiliencia en el siglo XXI. En M. Munist, N. Suárez Ojeda, D. Krauskopf \& T. Silber (Comp.), Adolescencia y resiliencia (pp.19-36). Buenos Aires: Paidós.

Laspina, M. (1996). Concepto de espiritualidad en el contexto de la enfermería. Boletín Impulso, 6, 27-29.

Lee, M., Nezu, A. M., \& Nezu, C. M. (2014). Positive and negative religious coping, depressive symptoms, and quality of life in people with HIV. Journal of Behavioral Medicine, 37(5), 921-930. doi: 10.1007/s10865-014-9552.

Machuca, J. (2002). Resiliencia, una aproximación a la capacidad humana para afrontar la adversidad [Tesis de grado]. Bogotá: Universidad Nacional de Colombia.

Matrángolo, G., Paz, G., \& Simkin, H. (2015). Factores de la personalidad y su relación con la autoestima, la espiritualidad y la centralidad de los eventos traumáticos. Psocial, 1(2).

Mezzadra, J., \& Simkin, H. (2017). Validación de la Escala Abreviada de Afrontamiento Religioso Brief-RCOPE en el Contexto Argentino en estudiantes de confesión católica. Evaluar, 17(1), 18-28

Morera, A. (2007). Estrés, Vulnerabilidad y Resiliencia. Extraído el 25 Junio, 2011 de http://www.auroramorera.com/2007/05/07/ estrés-vulnerabilidad -y-resiliencia-i/.

Muller, M. (2008). Formación docente y psicopedagógica. Buenos Aires: Bonum.

Muñoz, A. (2004). Cuestiones Epistemológicas Relativas al Estudio Psicológico de la Vivencia Religiosa. Psykhe (Santiago), 13(1). http://doi.org/10.4067/S071822282004000100011

Navas C. y Villegas H. (2007). Espiritualidad y salud. Saberes compartidos, 1, 34-42.

Pargament, K. I. (1997). The Psychology of Religion and Coping: Theory, Research, Practice. New York: Guilford Press.

Phelps, A. C., Maciejewski, P. K., Nilsson, M., Balboni, T. A., Wright, A. A., Paulk, M. E., Prigerson, H. G. (2009). Religious coping and use of intensive life-prolonging care near death in patients with advanced cancer. JAMA, 301(11), 1140. doi: $10.1001 /$ jama.2009.341

Piedmont, R. L. (1999). Does Spirituality Represent the Sixth Factor of Personality? Spiritual Transcendence and the Five-Factor Model. Journal of Personality, 67(6), 9851013. doi:10.1111/1467-6494.00080

Piedmont, R. L. (2001). Spiritual transcendence and the scientific study of spirituality. Journal of Rehabilitation, 67(1), 4-14. 
Piedmont, R. L. (2004). Assessment of spirituality and religious sentiments, technical manual (1a ed.). Timonium, Maryland: Author.

Piedmont, R. L. (2004). Spiritual Transcendence as a Predictor of Psychosocial Outcome From an Outpatient Substance Abuse Program. Psychology of Addictive Behaviors, 18(3), 213- 222. http://doi.org/10.1037/0893- 164X.18.3.213

Piedmont, R. L. (2004). The Logoplex as a paradigm for understanding spiritual transcendence. Research in the social scientific study of religion, 15(1), 263-284. http://doi.org/10.1037/0893-164X.18.3.213

Piedmont, R. L. (2012). Overview and development of measure of numinous constructs: The Assessment of Spirituality and Religious Sentiments (ASPIRES) Scale. En L. J. Miller (Ed.), The Oxford Handbook of Psychology and Spirituality (pp. 104-122). Oxford: Oxford University Press.

Poseck, V., Baquero, B. \& Jiménez, M. (2006). La experiencia traumática desde la psicología positiva: resiliencia y crecimiento postraumático. Papeles del Psicólogo, 27(1), 40-49.

Principe, W. (1983). Toward defining spirituality. Studies in Religion, 12(2), 127-141.

Rodríguez, M., Fernández, M., Pérez, M., \& Noriega, R. (2011). Espiritualidad variable asociada a la resiliencia. Cuadernos Hispanoamericanos de psicología, 11(2), 24-49.

Rosmarin, D. H., Bigda-Peyton, J. S., Öngur, D., Pargament, K. I., \& Björgvinsson, T. (2013). Religious coping among psychotic patients: Relevance to suicidality and treatment outcomes. Psychiatry Research, 210(1), 182-187. doi: 10.1016/j.psychres. 2013.03.023

Sánchez, B. (2004). Dimensión espiritual del cuidado en situaciones de cronicidad y muerte. Surgen luces de esperanza para acoger mejor el reto de la totalidad, tras años de investigación en enfermería. Aquichan, 4(4), 6-9

Simkin, H. \& Cermesoni, D. (2014). Factores de la Personalidad, Espiritualidad y su relación con la Calidad de Vida. Calidad de vida, 11(1), 7-17.

Simkin, H. \& Etchevers, M. (2014). Religiosidad, espiritualidad y salud mental en el marco del Modelo de los Cinco Factores de la Personalidad. Acta Psiquiátrica y Psicológica de América Latina, 60(4), 265-275.

Simkin, H. (2016). El modelo circumplejo de la espiritualidad. Psocial, 2(2), 65-76.

Simkin, H. (2017). Adaptación y Validación al Español de la Escala de Evaluación de Espiritualidad y Sentimientos Religiosos (ASPIRES): la trascendencia espiritual en el modelo de los cinco factores. Universitas Psychologica, 16(2), 1-12. https://doi.org/10.11144/Javeriana.upsy16-2.aeee

Simkin, H., \& Azzollini, S. (2015). Personalidad, autoestima, espiritualidad y religiosidad desde el Modelo y la Teoria de los Cinco Factores. PSIENCIA. Revista Latinoamericana de Ciencia Psicológica, 7(2), 339-361.

Vallurupalli, M., Lauderdale, K., Balboni, M. J., Phelps, A. C., Block, S. D., Ng, A. K.... Balboni, T. A. (2012). The role of spirituality and religious coping in the quality of life of patients with advanced cancer receiving palliative radiation therapy. The Journal of Supportive Oncology, 10(2), 81-87. doi: 10.1016/j.suponc.2011.09.003

Villalba, Q.C. (2003). El concepto de resiliencia individual y familiar. Aplicaciones en la intervención social. Revista sobre Igualdad y Calidad de Vida, 12(3), 283-299.

Windle, G. (2011). What is resilience? A review and concept analysis. Reviews in Clinical Gerontology, 21, 152-169. DOI: 10.1017/S0959259810000420. 